\title{
LITTÉRATURE
}

\author{
MICHAŁ BAJER
}

Université Adam Mickicwicz, Poznań

\section{SUR CERTAINS ASPECTS DE LA CRISE DES VALEURS HEROÏQUES DANS LES TRAGÉDIES EN MUSIQUE DE PHILIPPE QUINAULT}

\begin{abstract}
A bstract. Bajer Michal, Sur certains aspects de la crise des valeurs hérö̈ques dans les tragédies en musique de Philippe Quinault [On Certain Aspects of the Crisis of Heroic Valucs in the Music Tragedies of Philippe Quinault]. Studia Romanica Posnaniensia, Adam Mickiewicz University Press, Poznań, vol. XXXl: 2004, pp. 3-22. ISBN 83-232-1353-4, ISSN 0137-2475.
\end{abstract}

The French music tragedy, created in the 70 . of the 17 th century, may be considered against a background of the tradition of the heroic literature of the first half of the century. However, the appearance of opera coincides with a general crisis of consciousness and one of the features of the crisis was the fall of the heroic myth. The aim of the dissertation is to take a closer look at the mechanisms of destruction of the hero, used in Philippe Quinault's music tragedies. Opera gradually eliminates the traditional vision of love, replacing it with the conception of a devastating passion. In taking the pattern of the hero's choice, appropriate for the heroic vision, it changes the configuration of accents by creating the type of the "pathological hero".

Longtemps, la notion d'héroïsme constituait un des problèmes cruciaux de la critique dix-septièmiste. Durant cinquante ans qui ont suivi la Deuxième Guerre Mondiale, seul l'héroïsme comélien a donné lieu à quatre monographies ${ }^{1}$; en 1973 , l'Université de Strasbourg a organisé le colloque consacré à la conception du héros

1 O. Nadal, Le sentiment de l'amour dans l'ouvre de Pierre Corneille, Gallimard, Paris 1948; S. Doubrovsky, La dialectique du héros, Gallimard, Paris 1963; A. Stegmann, L’Héroïsme cornélien. Genèse et signification, Armand Colin, Paris 1968 (2 vol.); M. Prigent, Le Héros et l'Etat dans la tragédie de Pierre Corneille, P. U. F., Paris 1986. Il convient de mentionner aussi l'étude de $\mathrm{P}$. B énichou intitulée Le héros cornélien qui ouvre le livre Morales du grand siècle, Gallimard, Paris 1948 ainsi que certains des articles publiés dans le volume: Pierre Corneille, Actes du Colloque de Rouen (1984), P. U. F., 1985. 
dans la littérature de la première moitié du grand siècle ${ }^{2}$. Malgré le parallélisme temporel avec le structuralisme, les termes de la structure, du système etc. n'apparaissent pas dans les travaux d'Octave Nadal, André Stegmann ni Maurice Mongrédien. Cependant, dès l'ouvrage du premier d'entre eux, une certaine façon de penser dans les catégories du système se laisse noter. "On pressent, affirme l'auteur du Sentiment de l'amour dans l'cuvre de Pierre Corneille à propos des recherches cornéliennes antérieures, une erreur (...) [dans l'interprétation des] mots [devoir, passions], estime, vertu, générosité. N'entend-on pas sous ces mots l'idéologie particulière à notre temps, à nos façons de sentir, à nos moeurs? Or il arrive bien qu'elle corresponde dans quelques cas à l'idéologie entendue sous ces mots par les cornéliens, mais le plus souvent toutes les deux ne peuvent pas se recouvrir. (...) Cette interrogation nous a paru justifier les recherches qui suivent» ${ }^{3}$.

En simplifiant, on peut conclure que «tout ce qui a suivi», tout cet immense effort d'érudition a abouti à la reconstruction d'un certain système, dans lequel les notions clés de la littérature hérö̈que ${ }^{4}$ sont organisées dans un tout, où elles fonctionnent les unes par rapport aux autres, se définissent mutuellement, se renforcent. C'est à cette tradition déjà classique de la recherche que nous voulons nous référer, en appliquant certains de ses outils critiques à l'analyse des textes de Philippe Quinault.

Dans les années 1640 Mazarín a essayé d'introduire en France le théâtre musical italien. La nouveauté n'a pas plu aux parisiens. Ce sont Jean-Baptiste Lully et Philippe Quinault que l'on considère traditionnellement comme créateurs du véritable opéra français. En moins de vingt ans les deux auteurs ont fait jouer onze tragédies en musique ${ }^{5}$. Il est sûr qu'une telle création ne vient pas ex nihilo, comme elle ne se produit pas dans le vide. Déjà à l'étape des projets, l'opéra français était situé par rapport au système poétique classique au point d'en faire partie ${ }^{6}$; les premières tragédies en musique ont été considérées par leurs contemporains comme auvres littéraires ${ }^{7}$. Cadmus et Hermione, présentée en 1673, portait la qualification

${ }^{2}$ Les communications et les discussions ont été publiées dans le livrc Hérö̌sme et création littéraire sous les règnes d'Henri $V$ et de Louix XIII, Actes et colloques n ${ }^{\circ}$ 6, Klincksicck, Paris 1974.

${ }^{3}$ O. Nadal, op. cit., p. 286.

${ }^{4}$ Dans cette catégorie on retrouve un éventail très large des ccrits: les textes littéraires, philosophiques (moraux), historiques, politiques etc.

${ }^{5}$ Cc sont précisément ces textes-ci que nous analysons dans lc présent travail: Cadmus et Hermione (1673), Alceste (1674), Thésée (1675), Atys (1676), Isis (1677), Proserpine (1680), Persée (1682), Phaéton (1683), Amadis (1684), Roland (1685), Armide (1686).

${ }^{6}$ Le problème d'insertion des formes du théâtre lyrique dans la théorie classique des genres poétiques est décrit par Catherine Kintzler dans le livre La Poétique de l'opéra français de Corneille a Rousseau, Klincksieck, Paris 1993.

${ }^{7}$ C. Girdlestone, La tragédie en musique 1673-1750 en tant que genre littéraire, Droz, Genève 1973, p. 4-6. 
de tragédie en musique. Déjà par son nom générique l'opéra évoque donc la tradition littéraire classique. Il en est de même pour les thèmes. D'où vient la thèse que nous voudrions soutenir: l'opéra français peut être envisagé par rapport à la tragédie classique; en mettant en relief la figure du héros, la tragédie en musique renoue avec la tradition littéraire de la première moitié du siècle. Elle s'inspire de la vision héroïque dans la caractéristique du héros dans ses deux aspects: social et sentimental. Le héros - un individu exceptionnel capable d'actions glorieuses - est l'homme confronté à d'autres hommes, à la société. Tel est le sens de l'honneur nobiliaire. Comme l'écrit Octave Nadal: «On ne peut isoler l'honneur de la structure sociale. Formant un lien solide entre le héros et sa génération, il relève d'un savoir-vivre et de mœurs particuliers. (...) Cet honneur transmis par la coutume $(\ldots)$ rend cette élite sensible au scandale et à l'opinion, à la louange et à l'injure, aux décorations et aux disgrâces de la société» ${ }^{8}$. Avec le soin de sa renommée, le héros s'inscrit dans la sphère du public. Ceci ne veut pas dire qu'il soit pour autant exclu du domaine de l'intime. Chez les auteurs français de la première moitié du XVII ${ }^{\mathfrak{e}}$ siècle la vision héroïque intègre souvent la conception de l'amour élaborée dans les cercles précieux'. Ainsi le schéma de la rencontre d'un individu hérö́que avec les autres n'est-il complet que lorsqu'il comporte deux volets: l'homme face à la société et l'homme face à l'être aimé. La sensibilité du XVII ${ }^{e}$ siècle alimentée par le goût baroque pour les hyperboles et les antithèses est souvent celle du conflit: les concepts se heurtent, les valeurs s'excluent, les motivations s'abolissent. En ce qui concerne la psychologie héroïque, la possibilité du conflit entre le public et l'intime doit toujours être prise en considération. Même plus, il semble qu'à une certaine étape un tel conflit est inévitable. L'opéra, en reprenant ce dilemme marque ses liens avec la tradition hérö̈que.

Toutefois le raisonnement qui suit va s'appuyer également sur une opposition: nous allons opposer la tragédie en musique à cette même tradition littéraire dans laquelle elle puise. Une telle opposition n'est-elle pas abusive? Sur quoi se fonde-telle? Le premier argument est d'ordre chronologique: l'opéra apparaît relativement tard dans l'histoire de la littérature du XVII ${ }^{\mathbb{e}}$ siècle. Le deuxième argument découle du premier, mais il appartient au domaine de l'histoire intellectuelle. Les succès de l'opéra coïncident avec ce que, depuis Paul Hazard, on a pris l'habitude d'appeler «la crise de la conscience européenne» ${ }^{10}$. Une des composantes de cette crise était le crépuscule de l'idéal héroïque ${ }^{11}$. Entre Corneille et l'entourage du comte de

\footnotetext{
${ }^{8}$ O. Nadal, op. cit.

${ }^{9}$ Ibidem; S. Kevorkian, Le thème de l'amour dans l'auvre romanesque de Gomber-ville, Klinckisicck, Paris 1972.

${ }^{10} \mathrm{P}$. Hazard, La crise de la conscience européenne 1680-1715, Fayard, Paris 1961.

${ }^{11}$ P. Bénichou, La démolition du héros, in: Morales du Grand Siècle; A. H. Lévi, La disparition de l'héroísme: étapes et motifs, in: Héroïsme dans la littérature..., op. cit.
} 
Belin: les hommes de la première moitié du siècle et Quinault dans les années 1680 , le monde d'idées a changé de face. La philosophie, la vie sociale (le circuit littéraire ${ }^{12}$ ), les mœurs ne sont plus les mêmes. La figure du héros partagé entre ses devoirs envers la collectivité d'un coté et ceux envers la femme aimée de l'autre, est le point de départ de toute une conception du rapport entre l'honneur et l'amour qui réfléchit, semble-t-il, les caractères essentiels de la mentalité d'une partie de la société française à deux moments importants de son histoire. L'analyse des formes que prend ce conflit dans les tragédies en musique de Quinault devra rendre compte de la profondeur des transformations, voire des ruptures qui marquent l'histoire intellectuelle (l'idéologie ${ }^{13}$ ) du XVI ${ }^{e}$ siècle. L'objectif du présent article ne sera pas de démontrer que la tragédie en musique, comme la grande partie de la littérature de la fin du XVI ${ }^{\mathrm{e}}$ porte les symptômes de la crise universelle des valeurs. L'accent se déplacera du «pourquoi» vers le «comment» des choses. Nous essayerons de décrire les mécanismes qui font que la tragédie en musique de Quinault représente l'univers éthique «en panne». En fin de compte, nous voudrons saisir le spécifique du fonctionnement de ces mécanismes: en mettant en scène un nouveau type du héros (le héros pathétique), les textes de Quinault participent à l'effondrement des valeurs traditionnelles d'une façon qui leur est propre.

\section{DU HÉROS TRIOMPHANT AU HÉROS PASSIONNEL}

Il n'est rien de si beau que les nœuds de l'Amour,

Quand ils sont formés par la Gloirc (Thésée, I, 3).

Le point de départ des héros de la tragédie en musique est la situation de l'accord complet et calme entre ce qu'ils appellent leur amour et leur gloire. Ce modèle exclut la dissonance. Cadmus et Hermione, Thésée et Eglée, Alceste et Admète, Persée et Andromède comme Amadis et Oriane aiment d'amour sensuel (la beauté les caractérise tous) mais aussi grave, capable de faire face aux problèmes sérieux, capable, enfin, de sacrifices.

Hé bien! je périrai, si le destin l'ordonne;

Je veux délivrer Hermione;

Et si je l'entreprends en vain,

Je ne saurais périr pour un plus beau dessein (Cadmus et Hérmione, I, 1).

${ }^{12}$ R. Chartier, Lectures et lecteurs dans la France d'Ancien Régime, Seuil, Paris 1982; R. Chartier, Culture écrite et société, Albin Michel, Paris 1996; G. Dotoli, Littérature et sociésé en France en XVII siècle, Niazet, Paris 1987; J. Mesnard, La culture du XVII s., P. U. F., 1992.

${ }^{13}$ Dans le sens que donne à ce mot Paul Bćnichou dans l'entretien avec Tzvetan Todorov intitulé La littérature comme fait et valeur: l'idéologie y est comprise «en tant qu'activité de l'esprit posant des valeurs (...) une des facultés fondamentales de l'humanitè». 
Le dévouement amoureux trouve son incarnation la plus accomplie dans la figure emblématique d'Alceste qui, d'après la légende, accepte de mourir pour sauver son époux. Son amour fidèle obtiendra la sanction presque religieuse: les paroles de Proserpine sont la citation de Cantique des Cantiques:

Il faut que l'amour extrême

Soit plus fort

Que la mort (Alceste, IV, 5).

Heurté à des obstacles, l'amour cause de la peine. Les héros évoquent avec nostalgie l'indifférence passée:

J'aime l'heureuse paix des cœurs indifférents;

Si leurs plaisirs ne sont pas grands,

Au moins leurs peines sont légères (Atys I, 1) ${ }^{14}$.

Cependant, même arrivés au comble de la souffrance, ils ne maudissent jamais l'amour. Cet état d'âme qui se complaît dans sa propre souffrance amoureuse trouve la définition dans les paroles d'Hermione:

Laissez-moi ma douleur, j'y trouve des appas (Cadmus..., 6, II).

Le sacrifice de l'amour qui renonce à lui-même (pour sauver l'objet aimé) va être le moment culminant d'un bon nombre d'histoires d'amour présentées sur la scène d'opéra. Hermione, voyant son amant menacé, s'écrie:

Ah! Cadmus pourquoi m'aimez vous?

Pourquoi vouloir chercher une mort trop certaine?

(...)

J'aurais mieux aimé votre haine (Cadmus ..., 4, II).

Ainsi agiront Eglée et Andromède. Dans le dénouement d'Alceste, Alcide cède à Admète la reine qu'il aime, qu'il a sauvée et qui lui est due mais qui ne l'aime pas de son côté. Cet «héroïque effort» d'un demi-dieu qui renonce à son amour va être acclamé comme la plus éclatante de ses victoires ${ }^{15}$.

Finalement, la souffrance est une épreuve. Les sacrifices, la douleur etc.: tout se fond dans la gratification finale qui en est le prix. Il semble que sur ce point les premiers textes de Quinault s'accordent avec la conception de l'hérö̈sme élaborée dans la première moitié du siècle. Un de ses éléments caractéristiques était, comme on l'a dit, la présence du conflit sur la ligne: l'honneur - l'amour. Or, selon Paul Bénichou, il ne s'agit là que d'une fausse alternative, ses deux membres n'étant en réalité que deux aspects inséparables du même phénomène, à savoir l'orgueil noble. «ll serait facile de montrer que Comeille (...) confond l'honneur et le cour plus profondément qu'il ne les oppose, et que tout mouvement du drame va, chez lui, de

\footnotetext{
${ }^{14}$ Mais l'indifférence d'Atys est affectée.

${ }^{15}$ Alceste, $\mathrm{V}$, scène dernière.
} 
la division passagère de l'âme à la conscience retrouvée de son unité» ${ }^{16}$. Le même trait est souligné cinquante ans plus tard par Michel Prigent: «En s'acquittant d'une double dette envers l'honneur et envers son père, Rodrigue commençait de mériter Chimène. Preuve (...) qu'il ne faut pas opposer l'amour et héroïsme qui reposent sur les mêmes principes»" ${ }^{17}$. Dans cette perspective, le trait majeur du héros classique est l'unité de son caractère ${ }^{18}$.

Le problème de la relation entre la gloire et l'amour est posé explicitement dans le texte d'Andromède de Corneille. La protagoniste a oublié Phinée qu'elle avait aimé mais qui l'a quittée, en proie au monstre marin. Elle se prépare à épouser Persée. Ce changement, est-il profond? Les nymphes, compagnes d'Andromède s'engagent dans la discussion. Aglante parle des «flammes» et de "principes secrets d'aimer et de haïr»:

Nous en voyons au votre [cœur] en cette haute estime

Que vous nous témoigniez de ce bras magnanime; (...)

Et cette haute estime.

N'était qu'un beau degré pour monter à l'amour (Andromède, v. 1150-1158).

Aglante développe le modèle dialectique des relations entre la gloire et l'amour: l'une est la condition nécessaire de l'autre. Une autre nymphe s'oppose:

Si je puis toutefois vous le dire sans crime,

C'est hasarder beaucoup que croire entièrement

L'impétuosité d'un si prompt changement (lbid., 1159-1161).

Le problème reste ouvert et le colloque est interrompu par l'arrivée de Phinée. C'est lui qui, en posant le même problème, établira entre les deux valeurs la relation d'opposition:

Elle a cru tout devoir à son libérateur,

Mais souvent le devoir ne donne pas le cœur (Ibid., 1457-1458).

Toutefois si cette opposition est établie ce n'est que pour être tout de suite démolie par le discours d'Andromède elle-même qui clôt définitivement la controverse en établissant un lien étroit entre «aimer» et «mériter»;

C'était aux yeux du monstre, au pied de ce rocher,

Que l'effet de ma foi se devait rechercher; (...)

Cessez de m'envier ce que le ciel m'envoie,

Et souffrez que je tache enfin à mériter,

Au refus de Phinée, un fils de Jupiter (Ibid., 1577-1586).

${ }^{16}$ P. B énichou, op. cit., p. 48.

${ }^{17}$ M. Prigent, op. cit., p. 40.

${ }^{18}$ Même si cet accord ne se fait nullement d'une façon indolore. André Stegmann parle de la «dimension tragique de l'héroïsme cornélien». Nous reviendrons sur ce problème dans la dernière partie de cet article. 
Comme l'écrit Octave Nadal: «Dans ce théâtre héroïque, l'amour est lui-même héroïque; l'estime de la personne (...) accompagne toujours l'amour véritable. (...) Cela ne veut pas dire que l'amour soit fondé sur l'estime [on peut estimer sans aimer pour cela]; mais qu'il se nourrit d'estime réciproque: c'est un amour glorieux» ${ }^{19}$. Cette situation de l'amour glorieux peut être résumée par la formule suivante: «A aime B qui mérite d'être aimé; A mérite d'être aimé à son tour».

Face au dilemme la gloire - l'amour, Atys et Sangaride, Io (Isis), Roland, Angélique et Médor (Roland), ainsi qu'Armide, vont tout sacrifier à leur amour. Ceci se fera en raison de l'introduction au cœur de ce dilemme de la conception de la passion que Paul Bénichou appelle «naturaliste»: «un entrainement violent, étranger à tout sentiment de la dignité, et plus enclin à faire abdiquer le moi qu'à l'exalten ${ }^{20}$. Quant aux points caractéristiques de cette conception, nous voulons insister sur le pouvoir de la passion d'aliéner les héros, de les arracher à leurs états habituels, de les transformer. C'est le coup de foudre et la maladie ${ }^{21}$, enfin c'est la force fatale 22 .

La pièce où l'état du héros confronté à la passion destructrice est analysé avec le plus de netteté est Roland, l'adaptation de Roland furieux d'Arioste. Roland, amoureux d'Angélique, abandonne ses occupations chevaleresques, «laisse ternir sa gloire», pour s'adonner tout entier à servir sa passion. Rebuté, il finira par perdre sa raison. Angélique qui est princesse et qui devrait se lier avec le héros et le neveu de Charlemagne est, elle aussi, tourmentée par un amour déshonorant pour un beau captif africain, Médor. Finalement Angélique s'enfuit avec son amant. Quant à Roland, assisté par les forces surhumaines, il sera guéri de son délire.

L'épopée d'Arioste est un texte capital dans l'histoire de la conception héroïque de l'homme à l'âge classique. On peut s'attendre naturellement à ce que ses adaptations sur le terrain de la littérature française du XVII siècle réfléchissent naturellement ses avatars. Cinquante ans avant la tragédie en musique de Quinault, la folie de Roland a été le sujet de la tragicomédie de Mairet. La pièce a vu le jour dans le milieu aristocratique du comte de Belin.

Ce qui frappe dès le début dans le rapprochement des deux pièces est un très grand nombre de différences, tout d'abord formelles. Certaines d'entre elles se laissent aisément expliquer par les lois de deux types du théâtre: parlé et lyrique. La tragédie en musique introduit par exemple les scènes de divertissement absentes de la pièce correspondante. L'intrigue de Roland Furieux garde de l'original italien toute une histoire autonome, sans lien évident avec l'action principale (l'histoire de Zerbin et Isabelle). Cette intrigue n'apparaît pas dans l'opéra qui, par son essence, se caractérise par une économie verbale poussée. Et pourtant les différences les plus importantes ne relèvent plus des particularités des genres. Il s'agit là, principale-

${ }^{19}$ O. Nadal, op. cit., pp. 290-295.

${ }^{20}$ P. Bénichou, op. cit., p. 22.

${ }^{21}$ La métaphore de l'amour-maladic apparaît dans Roland. Nous citerons ce passage par la suite.

${ }^{22}$ Une telle conception est formulée dans Roland (1, II); Phaéton (I, 2). 
ment, de certains bouleversements de l'ordre temporel que nous analyserons par la suite. D'une façon générale, on peut dire que les deux auteurs mettent les accents sur les élément divers de la même action. Nous avançons l'hypothèse selon laquelle les divergences entre ces deux élaborations du même sujet découlent du changement essentiel de la manière de penser l'idéologie héroïque. On verra par la suite que les images du héros présentées dans les deux textes non seulement ne peuvent pas être identifiées, mais elles restent en une opposition marquée.

Les innovations chronologiques introduites par Quinault concernent la double série d'épisodes concentrés autour de trois personnages principaux. Deux scènes durant lesquelles Roland se rend compte de l'infidélité d'Angélique possèdent strictement la même structure dramaturgique qu'elles avaient chez Mairet. Au cours de la première, le chevalier retrouve les inscriptions laissées par Angélique et Médor en témoignage de leur bonheur. Dans la deuxième, il apprend, par la bouche des bergers rencontrés par hasard, les détails de l'affaire. Seulement, si chez Mairet les deux scènes ouvrent la pièce (ce sont, respectivement, la scène 1 et 2 du premier acte), chez Quinault elles closent la tragédie (scènes 2, 3, 4 et 5 du quatrième acte; le cinquième acte, dans les deux textes, n'étant qu'une sorte d'épilogue). La deuxième modification concerne les personnages d'Angélique et de Médor. Chez Mairet, dès le premier moment ils apparaissent comme des amants heureux dont la passion a obtenu la sanction du mariage:

Sa fièvre s'esteignit, la mienne s'alluma

Dans mon sang amoureux que son feu consuma,

Jusques-là que mon mal eut tant de violence

Qu'il força ma pudeur à rompre le silence,

En fin ce beau vainqueur ne fut pas si cruel

Qu'il ne bruslat pour moi d'un brasier mutuel;

Hymen après ccla, qui rend tout légitime,

Nous permit de gouster des voluptez. sans crime (Roland furieux, II, 3).

La fièvre, l'inquiétude, les aveux difficiles: tout appartient au plus-que-parfait du récit; dans le présent de la scène tout est calme et le soin unique des deux époux est de chercher à éviter les persécutions de Roland importun. Chez Quinault par contre, dès les premiers mots, nous sommes introduits au cour de la lutte («Funeste amour, fierté cruelle, $/$ Ne cesserez vous point de tourmenter mon cœur» I, 1):

Mon ccur était tranquille, \& croyait toujours l'être,

Quand je trouvai Médor, blessé, près de mourir:

La pitié dans ce lieu champêtre

M' arrêta pour le secourir:

Le prix de mon secours est le mal que j'endure;

La pitié pour Médor a su trop m'attendrir;

Ma funeste langueur s'augmentait à mesure

Qu'il guérissait de sa blessure,

Et je suis en danger de ne jamais guérir (Roland, I, 2). 
La lutte continue, les sentiments restent encore secrets. L'action des deux premiers actes est dominée par la progression de la passion impossible, paraît-il, entre la princesse et le simple soldat. Rien n'a été dit ni accompli et les personnages demeurent à l'état de la suspension insupportable. Marquée par les tentations de rupture de la part des héros, l'histoire aura son point culminant dans la scène des aveux amoureux échappés quasi inconsciemment (II, 4).

Il convient de réfléchir à présent sur les conséquences dramaturgiques de ces modifications. En écartant la scène de la désillusion du début, à la fin de l'intrigue, Quinault fournit à Roland un mobile absent du texte de Mairet: l'espoir. Partant il parvient à maintenir la progression psychologique et dramaturgique là où son prédécesseur se voit réduit à multiplier les horreurs de la furie ${ }^{23}$. En déplaçant le moment de la naissance de l'amour entre Angélique et Médor du récit à l'action proprement dite, il en fait "un événement théâtral» au sens plein du mot. Les personnages agissent. Il faudra examiner maintenant les particularités de cette action.

La princesse est tourmentée par la conscience de l'indignité de sa passion. Elle balance ( Ah ! Que mon Cœur est agité! / L'Amour y combat la fierté; / Je ne sais qui des deux l'emporte» I, 1). Comme Hermione de Racine, pour prévenir la faiblesse qu'elle sent imminente, elle recourt à l'appui de sa confidente ${ }^{24}(\ll \mathrm{Ne}$ me déguise plus rien; parle, je te l'ordonne / Représente à mon Cœur la honte de son choix» I, 1). Elle décide de gratifier les services amoureux de Roland («Je songe, autant que je le puis, / A sa rare valeur, à son amour extrême» I, 1) et éloigne Médor. Au début du deuxième acte nous retrouvons Angélique avec sa confidente au milieu de la forêt où elles se sont rendues à la recherche de deux fontaines magiques «Dont l'une fait aimer et l'autre fait haïr» (II, 1). Angélique, résolue à boire à la fontaine de la haine, succombe pour la dernière fois:

Non, je ne cherche plus la fontaine terrible

Qui fait d'un tendre amour une haine inflexible:

C'est un recours cruel, je n'y puis recourir. (...)

Par ce remède affreux, je ne veux point guérir;

Je consens plutôt à mourir (Roland, II, 1).

Le même mouvement se laisse noter dans le comportement de Roland. Méprisé une fois de plus, il pense triompher de son amour («C'en est fait, pour jamais mes liens sont rompus» II, 2). Quelque temps après, il revient en mendiant «la douceur funeste» de revoir Angélique. Finalement il avoue toute I'humiliation de sa dépen-

${ }^{23}$ Le comble de l'horreur est atteint au moment où Roland dévore la dépouille crue d'un fan de biche chassé auparavant par Médor: «Ce petit animal fit comme une barrière / A ce Tigre affamé qui demeura derrière / Pour en faire un repas' tout sanglant et tout cru / Au juste étonnement de mes yeux qui l'ont veu; / Ainsi j'ay pris le bois, ainsi je dois ma vie / A ce même animal a qui je l'ay ravie».

${ }^{24}$ Dans la première scène de l'acte II d'Andromaque. Hcrmione ordonne à Cléone: «De tout ce que tu vois tâche de ne rien croire; / Crois que je n'aime plus, vante-moi ma victoire; Crois que dans son dëpit mon curur est endurci, Hélas! et s'il se peut, fais-le-moi croire aussi». 
dance ( Je vous verrai toujours impitoyable; / Mais le plus grand des maux est de ne vous voir pas» 2, III). L'amour de Roland est une torture parce que - selon les critères de la morale héroïque (et Roland est le premier des chevaliers) - il est un cercle vicieux. Le héros le découvre. Cet amour est absurde parce que son objet ne le mérite pas:

Quelle honte à mon cœur d'être cncor si fidèle!

Pourquoi vous trouve-je si belle?

Non, avec tant d'attraits, si charmants \& si doux,

Vous ne méritez pas, cruelle!

L'amour que j'ai pour vous (Ibid., III, 2).

Il est encore absurde par ce que le sujet qui l'éprouve ne mérite pas d'être aimé à son tour (dévoré par la passion destructrice, Roland n'accomplit plus d'actions héroïques et est dépourvu de sa valeur en tant que chevalier):

Je pardonne au mépris du reste des humains;

Je l'ai bien mérité, j'aurais torl de m'en plaindre.

J'abandonne ma gloire et la laisse ternir;

Je chéris le trait qui me blesse;

De mon égarement je ne puis revenir (Ibid.).

Si la situation de l'amour glorieux peut être résumée par la formule: «A aime B qui mérite d'être aimé; A mérite d'être aimé à son tour» nous pouvons dire que l'amour de Roland est vide, parce qu'il est vide à ses deux pôles (l'objet ne mérite pas qu'on l'aime; le sujet ne mérite que le mépris). Le cercle se referme: Roland aime et veut être aimé mais ne peut pas l'être car... il aime et ainsi de suite, da capo al fine.

\section{Eh! Pourquoi tant souffrir? pourquoi}

N'aurai-je pas pitié de moi? (lbid.).

C'est maintenant qu'il convient de réfléchir sur le sens des quelques vers qui closent la longue plainte de Roland. C'est précisément là que le paradigme de l'amour glorieux, tel qu'il a été décrit par Octave Nadal est dépassé. L'amour passion, tout destructif, tout dévalorisant qu'il est, ne trouvant sa raison d'être ni dans son sujet ni dans l'objet, se légitime par lui-même:

Je pardonne au mépris de reste des humains

(...)

Mais vous qui causez ma faiblesse

Est-ce à vous de me punir? (Ibid.).

Possédé par la passion-maladie, le neveu de Charlemagne renonce à sa gloire et, finalement à sa dignité. La tragédie en musique suit les étapes de cette dégradation du héros qui, du chevalier, devient la figure hyperbolique d'un «amoureux» passionnel réduit à sa soif et sombrant dans la folie. 
Nous avons parlé de l'activité des héros de Quinault. Il est vrai que beaucoup d'événements, chez Mairet tout simplement racontés, quittent dans l'opéra le passé accompli du récit. Il est vrai que les personnages agissent. Cependant, il s'agit d'une action étrange. A y voir de près, l'activité des héros n'en est pas une que dans la mesure où elle les conduit à prendre conscience de l'impasse. La structure dramaturgique de cette tragédie en musique débouche sur le paradoxe, car ce qui est la matière de toute action est l'impuissance même d'agir. Ce sont: l'incapacité dans laquelle sont les héros de s'opposer à la force qui trouve sa source en eux mêmes mais qu'ils ne comprennent pas, leur inaptitude à concilier les exigences contraires, qui règlent la succession des faits. Sur le plan d'idées, ce sentiment de se retrouver sur une fausse piste correspond directement à la crise des valeurs hérö̈ques. Tout ce qui avait été dit permet déjà d'établir une opposition préliminaire entre les deux textes: dans la tragédie en musique de 1685 l'amour d'Angélique et Médor est douloureux, fait naître le déchirement. Le même amour dans la tragicomédie de 1635 est serein et apaisé. Ce calme n'est donné ni d'une façon arbitraire ni naturelle; tout au contraire, il est le fruit de toute une stratégie argumentative complexe.

La mésalliance est une atteinte mortelle à la gloire de la personne d'une condition supérieure: tel est le sentiment qui anime les reines cornéliennes (Cléopâtre, Rodogune, Viriate, Sophonisbe, Thomire), telle est la logique sociale. Tel est, enfin, le point de départ du raisonnement des personnages de Mairet. Dans la scène 1 de l'acte I, Roland méditant sur l'infidélité d'Angélique l'imagine dans les bras de «l'indigne amant», d'un «brutal qui lui tient un discours à son honneur fatal». Le scandale de la passion qui unit la future reine à l'amant sans gloire: voici l'optique qui ouvre à la pièce de Quinault la perspective tragique et qui met en marche le cercle vicieux décrit plus haut. Cependant, dans la structure de Roland Furieux, un tournant s'annonce. En évoquant la virginité d'Angélique (le problème de la bienséance dans le texte de Mairet est un problème à part), Roland parle de «cette fleur dont le prix gardera de vieillir / La gloire de l'amant qui la pourra cueillin» (I, 1). Le renversement est d'une grande importance. Non seulement la princesse amoureuse n'est pas exclue du système des valeurs héroïques, mais elle y introduit l'amant obscur. «L'indigne», «un brutal» devient un amant glorieux. La grandeur a la force d'anoblir, pour ainsi dire, par ricochet; elle est contagieuse par le biais de l'amour. Dès le début, Mairet entrevoit donc la possibilité d'éviter l'impasse; de concilier la passion avec la vision héroïque. Le plein succès de cette entreprise est finalement possible grâce à deux circonstances: la royauté future de Médor d'une part; son hérö̈sme passé de l'autre.

Devenant l'époux d'Angélique, Médor accédera au trône de Cathay:

Certes parmy les fleurs de nostre solitude,

J'ay toujours cette épine et cette inquiétude,

Qui pour vostre respect me va sollicitant

De mettre nostre amour dans un trône éclatant. (...) 


\section{Et (...) je n'aurai point le repos que j'espère}

Que vous n'ayez remply le trône de mon père (Roland furieux, I, 3).

Angélique aime, pour sa beauté, un homme que le rang social rend indigne d'elle. Puisque l'amour d'Angélique fera de son amant un roi, c'est dans cette beauté même, inspiratrice de l'amour, que gisent les germes de la royauté future de l'époux. Sa royauté est donc la conséquence naturelle de sa beauté, comme elle en est le complément:

C'est là que cet esclat qui suit la royauté

Doit redoubler en vous celuy de la beauté,

Et que ces doux regards, ce ris et cette grâce

Seront accompagnez d'une sénate audace:

$O$ qu'il sera beau voir ces yeux ct ces cheveux

D'où sortent tant d'esclairs, d'où pendent tant de vœeux;

Ceux - cy par la couleur que la nature leur donne

Faire palir sur eux l'or de votre couronne,

Et ceux - là par le feu qu'astre leur a presté

Gagner sur vos brillants le prix de la clarté (lbid.).

En aimant la beauté du Beau Médor, Angélique aime la gloire de futur MédorMonarque. Le lien fragile entre l'amour et la gloire n'est pas compromis.

Un autre facteur important est le thème du courage de Médor. Dans le texte de Mairet il y a deux moments où celui-ci est mis en question. Ce sont d'abord les bergers qui, en parlant du héros absent constatent: «à dire vrai, Médor est plein de charmes, / Mais je ne pense pas qu'il soit fort propre aux armes» (I, 3). Ensuite c'est Angélique qui exprime la même crainte. Ce doute irrite Médor qui s'emporte:

Auriez vous donc encore

L'injurieuse peur qu'un rival ne m'outrage?

Non, non, comme l'Amour, j'ay le Cocur en bon licu,

Et vos embrassements qui m'on fait demi-Dieu

M'inspirent unc audace à nulle autre scconde

Qui me fait mespriser tous les dangers du monde. (...)

Assisté d'un regard de vos yeux amourcux,

Ny Roland, ny Renaud, ny de plus dangereux (...)

N'auront point de valeur qui ne cède à mes armes (Roland furieux, II, l).

Médor s'approche donc de César de Corneille où de Pyrrhus de Racine ${ }^{25}$. Ceci est d'autant plus possible que la vaillance du héros n'appartient pas uniquement au domaine des déclarations. Elle a été prouvée. Dans un long récit, copié fidèlement sur Arioste, Mairet rapporte toute une série d'événements hérö̈ques qui ont conduit à la blessure de Médor et, par conséquent, à sa rencontre avec Angélique. Médor, quoique d'une naissance obscure, est un guerrier glorieux, le favori de son prince:

${ }^{25}$ La Mort de Pompée, la tirade de César v. 1248-1258; Andromaque (1, 3) «PYRRHUS: Animé d'un regard je peux tout entreprendre / Votre Ilion encore peut sortir de la cendre". 


\section{ANGELIQUE:}

Je scay bien que mon nom, quand vous l'aurez appris,

Vous fera condamner le dessein que $\mathrm{j}$ 'ai pris;

Et que tout l'Univers apprenant mon histoire,

Dira que mon amour a fait tort à ma gloire (...) mais des qu'on aura veu

La grâce et la beauté dont mon Ange est pourveu

Surtout, quand pour sa gloire on aura connaissance

Du sujet dont ma flamme a tire sa naissance

Les plus rudes censeurs des amoureux appas,

S'ils n'approuvent mon choix, ne le blasmeront pas (Roland furieux, II, 3).

Le texte de Quinault élimine l'un après l'autre les éléments de la pièce de Mairet décrits ci-dessus. La constatation d'Angélique: «J'ai droit de faire un roi»; «Je veux mettre en vos mains une suprême puissance» sont les seuls moments durant les deux premiers actes où le sujet de la royauté future de Médor est soulevé. Si le lien entre l'amour et la gloire apparaît au troisième acte, lors de la cérémonie nuptiale ( $Q$ Qu'il est doux d'accorder l'amour et la grandeur»), il est tout de suite démoli par l'exhortation du même chœur quatre vers plus tard («Oubliez vos grandeurs plutôt que vos amours»). Le credo des deux amants est exprimé dans la scène IV de l'acte III:

Je ne veux que votre Coeur;

C'est l'unique empire

Pour qui je soupire.

C'est une négation de la conception développée par Angélique de la tragicomédie de Mairet. L'histoire des exploits de Médor n'y apparaît pas du tout ("Quand je trouvai Médor blessé...» - mais Angélique n'explique pas la cause de cette blessure). Médor y est «un simple soldat de la troupe d'Agramant» au lieu d'être un compagnon préféré du prince africain. Enfin, Angélique et sa confidente Thémire ont beau mettre en doute son courage, sa force etc., Médor l'écoutera sans s'en indigner. A suivre de près le code de la morale hérö̈que, Angélique de Quinault, contrairement à son homologue chez Mairet, peut être «blâmée» de son choix amoureux. Cet effet résulte de la construction même du texte dont le trait caractéristique est l'élimination rigoureuse de toutes les «circonstances atténuantes» présentes dans la pièce antérieure.

Mais l'élimination n'y est pas la stratégie unique. L'opéra comporte la scène de la rencontre entre Angélique et Roland (scène 2 de l'acte III) sans analogue dans la pièce de Mairet (ou ces deux héros ne se voient pas du tout). Pour assurer sa sécurité, Angélique fait semblant de céder aux prières de Roland. Celui-ci restant dupe, les deux amants s'enfuient désormais en liberté. Le motif du mensonge dont la victime est le chevalier crédule apparaît déjà chez Mairet. A cela près, toutefois, que dans la tragicomédie ce sont les simples bergers qui induisent Roland en erreur. 
Quant à Angélique, elle n'y est pour rien. L'importance de cette modification apparaît pleinement à la lumière d'un des textes théoriques classiques les plus connus, à savoir de la préface de Phèdre de Racine: «J'ai cru que la calomnie avait quelque chose de trop bas et de trop noir pour la mettre dans la bouche de la princesse qui a d'ailleurs des sentiments si nobles et si vertueux. Cette bassesse m'a paru plus convenable à une nourrice, qui pouvait avoir des inclinations plus serviles (...). Phèdre n'y donne les mains que parce qu'elle est dans une agitation d'esprit qui la met hors d'elle-même (...)». Nous avons affaire dans les trois cas au même type des relations entre le héros central d'une condition supérieure et les héros secondaires. Il suffit de remplacer le mot «calomnie» par «le mensonge». On voit donc que la tragédie en musique de Quinault va à contre courant de la tendance morale classique exprimée par les œuvres de Mairet et de Racine. C'est à la princesse même que l'on attribue toute la responsabilité d'un acte jugé indigne. Cet effet est d'autant plus fort que tout se passe sur le théâtre. L'amour se situe donc à l'origine de la décadence morale des personnages.

\section{LES HÉROS PATHOLOGIQUES}

Sa naissance divine contestée par le rival à la main de la princesse, Phaéton, le fils du dieu de Soleil, demande à son père la permission de conduire une seule fois son char. Dans sa gloire, il trouvera la mort.

Il est beau qu'un mortel jusqu'aux cieux s'élève,

Il est beau même d'en tomber (Phaéton, IV, 2).

Ce qui fait l'exceptionnel du personnage de Phaéton, n'est pas tellement son ambition de devenir ce qu'il n'est pas, que le mépris qu'il éprouve envers ce qu'il est à présent. Son ambition est toute négative: être ambitieux, être le fils de dieu c'est, selon Phaéton, ne pas accepter sa condition actuelle.

Le fils du dieu du jour doit être plus content

D'un trépas éclatant

Que d'une vie obscure (Ibid., 1, II).

L'ambition de Phaéton fait penser à un autre demi-dieu du théâtre tragique français, à savoir Achille d'Iphigénie de Racine. Nous voyons en lui la même volonté de dépasser les limites de l'humanité:

Les Dicux sont des nos jours les maîtres souverains;

Mais, Seigneur, notre gloire est dans nos propres mains.

Pourquoi nous tourmenter de leurs ordres suprêmes?

Ne songeons qu'à nous faire immortels comme cux-mêmes (lphigénie, I, 2) ${ }^{26}$.

${ }^{26}$ Phaéton dira: «Un Cauur comme le mien fait son destin luy-même» (II, 1). 
Nous y retrouvons aussi le même désir de rejeter ce qui est humain. Dans le dialogue avec Agamemnon, Achille fait le récit de sa naissance et du choix que les Parques lui ont laissé ( «ou beaucoup d'ans sans gloire / Ou peu de jours suivis d'une longue mémoire»). Dans les expressions comme "de la terre inutile fardeau» ou «la débile vieillesse» nous lisons le même dégoût de soi (dans le sens le moins pascalien que possible) qui va être la marque de Phaéton. On peut dire que la gloire des deux héros commence là où la vie se termine. Le moment central des deux pièces est celui où les héros sont amenés à confronter leur image de l'honneur avec l'exigence de l'amour.

Dans la révolte d'Achille contre Agamemnon on a vu quelquefois le symptôme de la dégradation du héros qui dégénère en un amant tendre, renonce à sa gloire et sacrifie tout à son amour. Ainsi, de nouveau, les deux valeurs semblent entrer en conflit, s'exclure mutuellement. C'est du moins ce que suggère Agamemnon:

Mais vous ne demandez, vous ne cherchez que Troie.

Je vous fermais le champ où vous voulez courir.

Vous le voulez, partez: sa mort va vous l'ouvrir (Ibid., IV, 6).

Ou bien la conquête de la Troie, ou bien la fidélité à la maîtresse - voici le raisonnement du chef des Grecs. Cependant, ici, comme dans Andromède, si une telle opposition est esquissée, ce n'est que pour être tout de suite dépassée dans la réplique d'Achille:

J'ai votre fille ensemble et ma gloire à défendre (lbid. $)^{27}$.

La fureur d'Achille vient de l'indignation du héros qui apprend que son nom a servi de prétexte pour attirer Iphigénie en Aulide:

C'est peu que de vouloir, sous un couteau mortel,

Me montrer votre cour fumant sur un autel:

D'un appareil d'hymen couvrant ce sacrifice

Il veut que ce soit moi qui vous mène au supplice?

Que ma crédule main conduise le couteau?

Qu'au licu de votre époux je sois votre bourreau? (Ibid., III, 6).

En protégeant la femme qu'on lui a promise, en s'opposant à ce que son nom soit souillé par la complicité dans un acte qu'il juge indigne, Achille montre bien que le geste dans lequel on a pu voir le dévouement aveugle du héros passionnel est, en réalité, celui du guerrier qui s'enivre de sa propre renommée. La morale hérö̈que reste intacte.

Phaéton est le fils de Soleil et de la nymphe Clymène. Il est amant de Théone mais, pour devenir roi, il aspire à épouser Libye, la princesse d'Egypte. Comme le

${ }^{27}$ Cette discussion entre Achille et Agamemnon reproduit le grand thème du conflit entre Rodrigue et Don Dic̀gue: ce dernier ne peux pas comprendre l'insertion de l'amour dans l'axiologic héroïque. 
remarque Pascal Paul-Harang, dès les premiers mots prononcés par ce héros nous savons que «1) il aime Théone; 2) il n'aime qu'elle; 3) il l'aimera toujours» et «Cette profession de foi ne sera jamais contredite» ${ }^{28}$. Pourtant:

Si Theone me parait belle,

La couronne est encore plus charmante à mes yeux (Ibid., I, 4).

C'est en vain que Clymène, la mère du héros, supplie son fils:

Vivez \& bornez vos désirs

Aux tranquilles plaisirs

D'une amour mutuelle:

Aimez, contentez-vous... (Ibid., 1, II).

«Vivre», «borner les désirs», «tranquilles», «plaisirs», «amour», «aimen», «se contenter»: voici la langue que Phaéton ne comprend pas et qu'il ne pourra jamais comprendre.

Quant à l'hymen de la princesse:

La seule grandeur avec elle me lie,

Et l'Amour ne s'en mêle pas (Ibid., III, 1).

Dans toute la tragédie il n'y a aucune scène de rencontre entre Phaéton et Lybie: la fille du roi n'est qu'un instrument. Pour Phaéton l'amour et la gloire sont inconciliables. Comme Achille de Racine, il est obsédé par l'idée de son honneur. Mais Achille, comme les héros cornéliens, a su insérer le sentiment dans l'ordre de la gloire. Phaéton a perdu cette aptitude.

Pour régir l'Univers les Destins m'ont fait naître

Si l'Amour m'en rendait le Maistre

Que mon bonheur serait charmant!

Pour être heureux parfaitement

Ce serait avec vous que je le voudrais être (Ibid., III, 1$)^{29}$.

D'après cet aveu, le bonheur parfait est inaccessible pour Phaéton. En choisissant une valeur au détriment d'une autre, il ne sera heureux que partiellement: quoi qu'il fasse il va souffrir, il ne sera jamais heureux et il s'y condamne consciemment.

L'idée du rejet de l'amour et de la frustration du héros qui en résulte s'accentue également dans la dernière tragédie en musique de Quinault: Armide (1686). Renauld, l'objet de l'amour d'Armide est le seul des chevaliers chrétiens à ne pas succomber aux charmes de l'enchanteresse. Trop engagée pour pouvoir se venger, Armide recourt à la magie qui fait de Renauld son amant. Une fois le charme rompu, Renauld partira:

\footnotetext{
${ }^{28}$ Pascal Paul-Harang, Phaéton ou la volonté de briller dans le livre joint au disque ERATO, 1993.

${ }^{29}$ Phaéton adresse ces mots à Théone qu'il quitte.
} 


\section{RENAULD:}

Armide, il est temps que j'évite

Le péril trop charmant que je trouve à vous voir:

La Gloire veut que je vous quitte;

Elle ordonne à l'amour de céder au devoir.

Si vous souffrez, vous pouvez croire

Que je m'éloigne à regret de vos yeux:

Vous régnerez toujours dans ma mémoire;

Vous serez, après la Gloire,

Ce que j'aimerai le mieux (Armide, $\mathrm{V}, 4$ ).

Renauld quittera Armide qu'il aime. Comme Phaéton, il décide de vivre avec le sentiment du manque.

Pour ces personnages nous voudrions proposer l'appellation des héros pathologiques (dans le sens de ce «qui est relatif à l'état de la maladie» ${ }^{30}$ ) en opposition au héros pathétique (dans le sens de ce «qui émeut vivement, qui excite les passions et les émotions vives» ${ }^{31}$ ).

Arrivés au sommet de leur grandeur, les héros de Corneille souffrent souvent. «La gloire cornélienne ne peut mener à la joie; le héros en prend noblement son parti», écrit Octave Nadal. La dimension tragique fait partie du mythe héroïque. Comme l'écrit Jean Chapelain dans la préface de La Pucelle: «(...) la vertu héroïque (...) ne regarde pas moins cette magnanimité qui fait souffrir les plus grands maux, que celle qui fait vigoureusement agir dans les entreprises les plus difficiles. Car, qui niera qu'un Régulus et un Socrate, un Paetus Thrasea et un Helvidius Priscus ne soit pas aussi bien des héros, par les peines qu'ils ont endurées avec tant de fermeté, qu'un Cyrus et un Alexandre, un Scipion et un Trajan, par les actions qu'ils ont faites avec tant de cœun ${ }^{32}$. Cependant, soit dans le triomphe, soit dans la chute, cette souffrance s'inscrit dans ce que nous avons signalé comme le trait distinctif de ces héros: le sentiment de la plénitude de leur épanouissement. Accompagnée de la lucidité pertinente, elle permet de scruter d'un coup d'oeil synthétique toute une vie, désormais érigée en symbole. C'est la souffrance d'un individu qui considère son triomphe ou son échec; elle ne met pas en danger l'unité du caractère classique. Elle est pathétique par la force des sentiments qu'elle est censée exciter, et parmi lesquels l'admiration occupe une place de choix. Ayant dépassé le stade du «sacrifice», les héros acquièrent la «singularité» et deviennent «exemplaires» ${ }^{33}$.

La situation des protagonistes de la tragédie en musique est différente. Dans Phaéton, Roland et Armide, le lien étroit entre l'honneur et l'amour disparaît, le mécanisme du sacrifice menant à l'apothéose est perdu. Phaéton, Roland et Renauld

\footnotetext{
${ }^{30}$ Le Grand Robert de la langue française.

${ }^{31}$ Ibidem.

${ }^{32}$ J. Chapelain, Opuscules critiques, Droz, Paris 1936, p. 263.

${ }^{33}$ M. Prigent, op. cit.
} 
sont victimes d'une impossibilité essentielle de cette unité classique du caractère, dans laquelle l'individu, écrasé ou triomphant, mais toujours devenu le centre de son monde et du monde en général, était capable d'embrasser les contraires, de les réduire au même principe. Si on essaie d'appliquer aux histoires de Roland et Renauld la triade proposée par Michel Prigent, on se rend compte de ce qu'elles ne dépassent pas le premier stade, qu'elles s'interrompent brusquement. La souffrance ne s'accompagne ni de la lucidité ni de la distance: nous quittons les héros en plein effort de sacrifice, au moment où ils sont trop plongés dans leur douleur pour pouvoir l'envisager avec du recul.

Pour se rendre compte de cette différence il est utile de revenir encore une fois au parallèle entre Roland furieux de Mairet et Roland de Quinault. A la fin des deux pièces la raison est rendue au chevalier infortuné. Alors, qu'est-ce qui permet de constater que les deux dénouements, pareils à la première vue, en réalité se contredisent?

Dans la tragicomédie, le secours vient de la part du parent de Roland, Astolphe, qui se rend au ciel d'où il ramène la fiole en cristal contenant le bon sens du paladin. Sur ce point Mairet suit directement l'original italien:

Vous ne savez peut-être pas ce qui s'est passé en ce moment en France, quoique vous en arriviez. Sachez donc que votre cousin Roland, pour n'avoir pas accompli la tâche qui lui avait été confiée, est puni de Dieu qui, Jorsqu'on l'offense, se montre plus irrité contre ceux qu'il aime le plus. (...) Ce Roland (...) a payé d'ingratitude les bienfaits du Scigneur. Quand son premier devoir élait de se consacrer au service du people fidèle, il l'a délaissé: une paìenne lui a inspiré un amour coupable (...) Pour le punir, Dieu a voulu qu'en proie à une folie furicuse, il courut à travers les campagnes (...). Son intclligence est tellement obscurcie et perdue qu'il ne reconnaît personne, qu'il ne se reconnaît lui-même. (...) Mais (...) la bonté divine a voulu que trois mois pussent suffire pour (...) expier [la fautc de Roland]. (...) C'est pour apprendre par quels moyens vous pourrez secourir Charlemagne et délivrer notre sainte religion du péril qui la menace que vous êtes venu chercher mes conseils; et, en vous permettant de faire un si long voyage, lc Rédempteur n'a eu d'autre but que de vous faire instruire par nous des moyens à employer pour faire recouvrir à Roland sa raison ${ }^{34}$.

De même Astolphe de Mairet agit «Suivant l'ordre du Ciel, reçu dans le Ciel même» $(\mathrm{V}, 3)$. Dans le discours qu'il tient en présence de Roland guéri nous retrouvons encore une fois tous les éléments soulignés dans l'épopée d'Arioste:

Et je suis trop discret pour vous faire le conte

D'un accident fameux par vostre propre honte;

Si Dieu qui veut de nous un juste repentir

Ne m'avait commandé de vous en advertir,

Pour avoir préféré l'amour d'une Payenne

Au salut de la France et de la foy Chrétienne,

${ }^{34}$ A rioste, Roland furieux, trad. Célestin Hippeau, Garnicr, Paris 1923, vol. 2, pp. 243-245. 
Du mépris du devoir vous êtes descendu

A la perte du sens que je vous ay rendu (Roland furieux, $V, 3$ ).

Dans la tragicomédie de 1630 , la furie est le châtiment que Dieu envoie au héros pour le manquement de fidélité. Elle s'intègre donc sans reste dans l'ordre héroïque. En étant sa négation symétrique temporellement et spatialement limitée ${ }^{35}$, elle est là pour renforcer ce qui l'entoure et n'a d'autre but que la correction du héros dans la perspective (jamais disparue) de sa gloire future. Roland a pu dévorer le gibier cru: tout ceci avait son objectif, était exemplaire (dans le sens dans lequel un exemple peut être négatif).

Dans l'opéra, l'intervention d'Astolphe se limite à implorer l'aide de la sage fée Logistille. Celle-ci, au moyen de la musique et de la magie (elle évoque les ombres des anciens héros) obtient la guérison de Roland. Comme la perspective transcendantale a été soigneusement éliminée de la pièce, l'aventure perd sa valeur de châtiment et correction calculés. Ainsi tout ce qui a précédé change de signification. Le délire de Roland d'un exemple devient une affaire intime; la souffrance n'a pas de valeur exemplaire.

Les finales de Roland et Armide justifient l'emploi de la métaphore médicale: les pièces finissent au terme du traitement hasardeux, mais elles ne montrent pas les fruits de la convalescence. La souffrance ne complète pas, elle mutile les héros, comme elle coupe le récit. Voici l'ultime avatar du mythe héroïque dans la tragédie en musique de Quinault.

Dans les onze tragédies en musique de Quinault placés dans leur contexte historique on peut aisément voir le signe du moment que Paul Hasard a appelé «la crise de la mentalité». Or, ce moment est précisément celui où l'écho des formes anciennes résonne encore suffisamment pour qu'il y ait le besoin de les réutiliser, réemployer. Cependant les changements culturels vécus par la société étant trop poussés, ces formes ne fonctionnent plus dans leur version primitive, intacte. En fin de compte, on se rend compte de l'abîme qui se dresse entre les mots (la gloire, l'amour) et l'univers des valeurs qu'ils véhiculent. Ce n'est qu'au niveau de ces premiers que la tragédie en musique continue la tradition héroïque: la vision du monde qu'elle finit par créer est, en réalité, tout différente. Le détournement du sens commence avec l'apparition du conflit insoluble entre le public et l'intime. Au lieu

${ }^{35}$ Cette limitation est mise en relief par toute une série d'événements à caractère symbolique liês aux armes de Roland. Au début de la crise de folie Roland rejette Durendal et se débarrasse de son armure (III, 1: «Mais jette cette épée et ces armes pesantes, / Tes mains a tous exploits sont assez suffisantesw). La pièce se termine par la promesse de les récuperer $(\mathrm{V}, 3)$. La folie se place donc entre deux actions quasiment rituelles: celle qui ouvrc (la perte des armes), celle qui clôt (leur reconquiste). Roland commet les actions indignes sans se servir de son épée ni de son armure. Ainsi les symboles de la chevalerie ne sont pas compromis par le contact avec le délire. 
de s'accorder (même douloureusement), les deux versants de la personnalité héroïque - l'exigence de la gloire et la tendresse envers l'être aimé - se heurtent. A partir de là, deux attitudes sont possibles. Premièrement, le héros peut laisser tomber le soin de sa renommée, s'abandonner tout à son sentiment: devenir un héros «dameret», pour nous référer à une formule de Boileau ${ }^{36}$. C'est le point commun de la plupart des attaques dirigées contre Quinault et la tragédie de son temps. Selon Boileau, le monde représenté par Quinault serait fade ${ }^{37}$. A propos du théâtre tragique contemporain, Saint-Evrémond écrit en 1689: «En effet, ce qui doit être tendre n'est que doux; ce qui doit former la pitié fait à peine la tendresse; l'émotion tient lieu du saisissement, l'étonnement, de l'horreur ${ }^{38}$. Cependant, ce qui a surtout attiré notre attention est l'autre aspect de la «mort du héros». Dans les personnages de Roland, Phaéton et Renauld nous avons vu les héros qui, confrontés au choix entre deux valeurs, se rangent toujours du coté de la gloire. Au prime abord, ils peuvent faire penser aux héros cornéliens de la première moitié du siècle. Cependant, comme nous l'avons vu, les ressemblances sont superficielles. Les protagonistes en question sont «les héros pathologiques»; leur héroïsme les enferme dans leur souffrance. Phaéton et Renauld sont frustrés. Or, le sentiment de frustration est, de par son essence, étranger à la psychologie héroïque qui se caractérise par l'épanouissement maximal. Il nous semble que beaucoup plus que le côté «dameret» de certains autres personnages, cette peinture du héros insatisfait est le vrai témoignage du crépuscule des valeurs héroïques.

\footnotetext{
${ }^{36}$ L'Art poétique, III, v. 118.

${ }^{37}$ Plusieurs passages de la Satire III sont dirigés contre le theâtre tragique de Quinault.

${ }^{38}$ St.-Evrémond, Sur les tragédies, Librairie Marcel Didier, Paris 1966.
} 